\title{
A extensão universitária na formação do cirurgião-dentista
}

\author{
Gabriela Pizzolatto*; Mateus José Dutra*; Daniela Jorge Corralo** \\ * Graduando (a) em Odontologia, Universidade de Passo \\ Fundo \\ ** Professora Doutora, Curso de Odontologia, Universidade \\ de Passo Fundo
}

Recebido: 13/11/2019. Aprovado: 25/07/2021

\begin{abstract}
RESUMO
As universidades têm se transformado ao longo dos tempos. O foco principal no ensino foi sendo ampliado com ações de pesquisa e de extensão. Esta vem ganhando cada vez mais espaço nas instituições de ensino superior, fazendo parte da tríade que sustenta a formação universitária. $\mathrm{O}$ objetivo desta revisão de literatura foi mostrar a importância da extensão universitária na formação profissional de cirurgiões-dentistas e para a sociedade. A estratégia de busca de artigos se deu por palavras-chave relacionadas à Odontologia e à extensão universitária em bases de dados eletrônicas, no período de agosto a outubro de 2019. A prática da extensão, em comunidades diversas, fortalece a compreensão dos estudantes quanto ao processo saúde-doença, permite conhecer a realidade econômica e social em diferentes cenários, permite vivenciar a rede de serviços públicos de saúde, assim como participar da prestação de serviços à população, tornando-os mais humanizados e capacitados a promover a saúde da comunidade em que estão inseridos. A extensão universitária tem um importante papel na formação profissional do cirurgião-dentista, colaborando para que atue de forma competente para contemplar as diversas necessidades da população, estimulando ações de promoção de saúde e informação à população em geral, que também se torna beneficiada com estas ações, e fortalecendo o compromisso da universidade com a sociedade.
\end{abstract}

Descritores: Extensão Comunitária. Odontologia Comunitária. Educação Continuada.

\section{INTRODUÇÃO}

Ao longo de sua existência, a universidade passou por numerosas transformações. Por muito tempo suas atividades eram focadas basicamente no ensino. Com o avanço científico, as atividades investigativas começaram adentrar as universidades, estimulando a pesquisa nas mais diversas áreas do conhecimento. Ainda durante o século XIX, as atividades extramuros se somaram à pesquisa e ao ensino ${ }^{1}$ constituindo a tríade que sustenta a formação universitária: o ensino, a pesquisa e a extensão. Esta última, por sua vez, se revela como um importante meio pelo qual a universidade leva assistência e conhecimento à 
população, por meio de atividades fora do ambiente acadêmico e que se constituem, ainda, como uma fonte para pesquisas, retroalimentando o ensino ${ }^{2,3}$.

O desenvolvimento e a ampliação destas atividades durante os últimos 40 anos teve como principais alavancas: (1) o rompimento do isolamento das universidades, aumentando a sua influência e notoriedade na sociedade, incentivado por alguns professores, com as mesmas motivações; (2) o desejo de mais experiências práticas e vivências formativas, por parte dos estudantes, durante sua formação; e, (3) a busca por conhecimentos que poderiam ajudar a população na resolução de seus problemas, levando informações que pudessem contribuir para a melhoria das condições da vida da sociedade ${ }^{1}$.

A extensão universitária deve ser integrada à matriz curricular e à organização da pesquisa. Ainda, deve ter uma estrutura de interdisciplinaridade, envolvendo questões político-educacionais, culturais, científicas, tecnológicas, promovendo transformações na própria instituição e na sociedade ${ }^{4}$. É um processo educativo, cultural e científico que articula o ensino e a pesquisa de maneira indissociável e propicia a relação transformadora entre universidade $\mathrm{e}$ sociedade. Sendo assim, essa relação não deve ser interpretada como unidirecional, na qual o conhecimento da universidade é difundido para a população, mas sim como uma via de mão dupla, em que os acadêmicos e professores também aprendem, colocam em prática e lapidam conhecimentos que já existiam previamente ${ }^{1,2}$.

A formação de profissionais da área da saúde mantém uma forte tendência tecnicista, que é focada na doença, em prejuízo à promoção da saúde. Dessa forma, muitos graduandos tornam-se profissionais que não entendem claramente como atuar nesta esfera. As atividades desenvolvidas na extensão universitária propiciam aos acadêmicos a oportunidade de conhecer a realidade econômica e social do local onde vivem e dos serviços públicos de saúde, assim como participar da prestação de serviços à população, tornando-se profissionais mais humanizados, que compreendem melhor o processo saúde-doença, e mais capacitados a promover a saúde da comunidade em que estão inseridos 5 .

Apesar das vantagens mencionadas, a extensão ainda é vista, muitas vezes, como uma atividade secundária, não tão valorizada $\mathrm{e}$ importante quanto o ensino e a pesquisa. A formação acadêmica, no entanto, não deve contemplar apenas os conhecimentos técnicos, deve oportunizar aos estudantes que aprendam a partir de suas experiências, colocando em pratica o que os é ensinado, aprofundando o conhecimento teórico e, em algumas situações, o ressignificando a partir da vivência extensionista. Ou seja, a extensão tem se tornado um meio de aprendizagem essencial para que os acadêmicos desenvolvam habilidades fundamentais para seu agir profissional $^{6,7}$. Desta forma, o objetivo desta revisão de literatura é apresentar a importância da extensão universitária na formação dos profissionais da área da Odontologia e discutir a sua importância na sociedade e o seu papel nas universidades.

\section{REVISÃO DE LITERATURA}

Foi realizada busca nas bases de dados eletrônicas SciELO e Google Acadêmico utilizando as palavras-chave extensão universitária e Odontologia. A seleção dos artigos foi feita entre agosto e outubro de 2019. Foram incluídos aqueles considerados relevantes para o tema em questão.

A complexidade da compreensão e do entendimento do processo saúde-doença exige que se reflita acerca da formação do profissional em saúde, a qual deve contemplar mais do que somente habilidades técnicas, que de fato são importantes para a prática profissional, porém não suficientes. É preciso ir além de uma visão instrumentalista e tecnicista do conhecimento, sendo papel das 
universidades a formação de profissionais comprometidos e responsáveis para com o mundo onde vivem ${ }^{8}$.

Apesar da compreensão da importância de uma visão social ampliada na formação dos profissionais, a abordagem de problemas sociais de saúde não causa grande entusiasmo e nem é tão valorizada entre os estudantes e professores. É usual que, na educação odontológica, conteúdos técnicos sejam reproduzidos nos ambulatóriosescola (clínicas), faltando aos profissionais da saúde uma sensibilidade social, fazendo com que criem uma visão distorcida da realidade da população e da rede de serviços de saúde, sendo de extrema importância que os acadêmicos tenham vivências em diferentes cenários de aprendizagem.

A ênfase na formação tecnicista não é, necessariamente, prejudicial, no entanto, não deve ser a única via de conhecimento. Um currículo que ainda prioriza os conteúdos técnicos é realidade na maioria das instituições e não dá ênfase aos aspectos socioculturais e epidemiológicos do processo saúde-doença. Este modelo de ensino, no qual o foco é a cura do problema e não a prevenção, não é efetivo no controle das doenças. É necessário que se forme um profissional como um todo, que não deve ser apenas um cirurgião-dentista, e sim um profissional cidadão, que seja comprometido com o desenvolvimento social ao seu redor ${ }^{2,6,8}$. As Diretrizes para a Extensão na Educação Superior Brasileira, estabelecidas em 2018 ${ }^{4}$, visam regulamentar as atividades acadêmicas de extensão integrando a sua prática aos componentes curriculares dos cursos. Desta forma, aposta-se que a partir da implementação das mesmas, o impacto positivo na formação dos estudantes possa ser reforçado e ampliado, alcançado um maior numero de futuros profissionais.

A formação deste profissional diferenciado e comprometido, é um desafio para as instituições de ensino. Para alcançar este objetivo formativo é imperativo que ele interaja efetivamente com a sociedade, para que se situe histórica e culturalmente, ou para referenciar sua formação técnica a problemas que um dia ele enfrentará ${ }^{9}$. A universidade tem o papel de formar profissionais capazes de lidar com as necessidades sociais, trabalhar em equipe, se comunicar, ser ágil ao enfrentar problemas, e que gerem ganhos para a civilização ${ }^{8}$.

Segundo Hennington $(2005)^{10}$, os programas de extensão universitária deveriam demonstrar a sua importância por meio da relação estabelecida entre a instituição e a sociedade, e assim se consolidando a partir da aproximação e troca de conhecimentos e experiências entre professoresestudantes-população, possibilitando o desenvolvimento de processos de ensinoaprendizagem baseados nas práticas cotidianas e correlacionadas com o ensino e a pesquisa, permitindo o confronto e o estudo das teorias aprendidas com o mundo real de necessidades e desejos.

$\mathrm{Na}$ área da saúde, atividades extensionistas assumem particular importância na medida em que se integram à rede assistencial e podem servir de espaço diferenciado para novas experiências voltadas à humanização, ao cuidado e à qualificação da atenção à saúde, além de ser uma ação facilitadora para que ocorram interações mais dinâmicas e verdadeiras entre os acadêmicos e a comunidade, trazendo benefícios para ambas as partes. Os alunos passam a ter uma visão mais humanista e social dos seus pacientes, aflorando o lado afetivo, mas não deixando de lado o aspecto técnico de sua profissão. O resultado é um ensino integral, onde pode ser colocado em prática o que foi aprendido em sala de aula, construídos novos conhecimentos, e proporcionando um conceito mais amplo do processo saúde-doença e do mundo onde vive, além de proporcionar o desenvolvimento da habilidade de comunicação, quesito importante para sua futura profissão, formando assim profissionais mais preparados para 
lidar com pessoas e para enfrentar o mercado de trabalho. Para a população, estes projetos proporcionam a oportunidade de aprender de forma gratuita, e melhorar suas condições de saúde, favorecendo mudanças sociais.

Quando um cirurgião-dentista ou qualquer outro profissional da saúde se restringe a trabalhar somente no campo biológico ou no trabalho técnico, reduz drasticamente a chance de oferecer saúde bucal para seus pacientes e para a comunidade, além disso, serviços comunitários motivam o acadêmico em relação à sua profissão, já que tornam muito mais gratificante o aprendizado, pois ele está contribuindo para melhorar a saúde e a qualidade de vida da população $^{6,11}$.

A extensão surgiu no século XIX na Inglaterra, com o objetivo de promover a educação continuada e atualmente oportuniza às universidades efetivar seu compromisso social" ${ }^{11}$. Segundo o Ministério da Educação e Cultura a extensão é um processo educativo, cultural e científico que une o ensino e a pesquisa e viabiliza a relação transformadora entre a universidade e a sociedade $^{9}$. Para que a universidade apresente relevância social, deve ser culturalmente engajada e comprometida com a resolução de problemas sociais, e não renunciar à sua relação reflexiva e ativa com o mundo ao seu redor. Dessa forma, a extensão universitária tem função de articular a comunicação entre a universidade e o seu meio, promovendo uma sociedade mais justa $\mathrm{e}$ igualitária ${ }^{8}$.

A universidade tem sua filosofia baseada em três pilares básicos, a docência, pesquisa e prestação de serviços, sendo o último o que permite uma relação direta com a comunidade ${ }^{6}$. Estas atividades são de caráter indispensável, possibilitando não somente o desenvolvimento de conhecimentos técnicos e científicos, mas também ajudando no progresso da sociedade ${ }^{12}$. De acordo com as Diretrizes Curriculares Nacionais para os cursos de Odontologia ${ }^{13}$, deve ser estimulado o conhecimento dos problemas presentes no mundo, a prestação de serviços específicos para a comunidade e programas de extensão abertos para a participação popular. Esta interação proporciona uma troca de saberes acadêmicos e populares, constituindo uma relação de intercâmbio de experiências, estratégia importante para que ocorra a transformação da realidade de saúde da população ${ }^{14,15}$. Neste contexto, se faz essencial que, com todos os mecanismos estruturais e científicos que as universidades possuem, ela amplie seus espaços de atuação para que ocorra uma troca com a sociedade, desempenhando seu papel fundamental, o de produzir conhecimentos ${ }^{6}$. Com isso, a universidade enquanto desenvolve projetos que atuam em benefício da comunidade, resgata seu comprometimento social ${ }^{18}$.

As atividades de extensão realizadas pelas universidades são uma forma de estimular o processo de mudança pelo qual a Odontologia tem passado. Grande parcela da população carente ainda acredita que seus problemas de saúde bucal, como a cárie, seguida por extrações e confecções de próteses, são uma fatalidade que não pode ser evitada, ou uma circunstância natural conectada à sua condição financeira. Somente uma prática odontológica com a filosofia de atuação fundamentada em promoção de saúde tem capacidade de mudar positivamente este pensamento. Diversos programas de extensão vêm sendo implementados nas instituições em todo país, com diferentes cenários de práticas e grupos etários e sociais. As atividades realizadas envolvem orientações, prevenção e até clínicas de extensão que oferecem tratamento curativo e preventivo para a população. Além disso, os trabalhos voluntários complementam a carga horária de formação ${ }^{6}$. O fortalecimento da relação entre sociedade e universidade propicia uma melhoria na qualidade de vida dos cidadãos, ao extrapolar os conhecimentos para outros ambientes além da sala 
de aula. Um dos principais objetivos dos programas de extensão é a mudança social, e se trata de um progresso da universidade em consonância com a comunidade $^{11}$.

Em 1988, o artigo 207 da constituição brasileira convocou uma reflexão por parte das universidades, para que estas articulassem as atividades de ensino, pesquisa e extensão de forma integrativa e complementar, vinculando a teoria e a prática. Desta forma, a pesquisa desenvolve e aprimora novos conhecimentos, que são transmitidos pelo ensino e extensão, fazendo com que estes três pilares da formação acadêmica se complementem e se correlacionem. A universidade, por meio da extensão, leva conhecimento e atendimento para a comunidade, que se torna uma fonte de pesquisa que, consequentemente, retroalimenta o ensino ${ }^{2}$. Um estudo realizado por Emmi et al., (2018) $)^{5}$, analisou a percepção de estudantes e de egressos da Faculdade de Odontologia de uma universidade do Pará, sobre as atividades extensionistas realizadas durante sua formação. Para realizar esta análise foram selecionados 36 portfólios das atividades extramurais entre os anos de 2009 e 2014. Complementarmente, foram entrevistados cinco egressos que estavam atuando no Sistema Único de Saúde (SUS), para que respondessem perguntas sobre como as atividades extramurais auxiliavam a sua atuação profissional, utilizando uma abordagem qualitativa. Os entrevistados citaram que as atividades extramurais contribuíram para que se tornassem profissionais mais humanos $\mathrm{e}$ éticos, que se preocupam com a saúde de seus pacientes, e que dão a devida importância para a promoção de saúde, além de lhes proporcionar um contato com a atenção básica de saúde e o sistema de saúde público, resultado das experiências vividas nas atividades extramurais, fazendo com que desenvolvam habilidades e qualidades imprescindíveis para o cirurgião-dentista.

Weiler et al. (2013) ${ }^{17}$ analisaram, com uma pesquisa quantitativa, os benefícios cívicos que os estudantes que participaram de um programa de extensão $(n=390)$ de apoio a jovens em situação de risco apresentaram comparando aos estudantes que não participaram do projeto $(\mathrm{n}=258)$. Os estudantes que vivenciaram as atividades extensionistas demonstraram ter maior confiança para atuar no serviço comunitário, desenvolveram habilidades pessoais e profissionais, e tornaram-se capazes de resolver problemas, além de desenvolver uma maior consciência política. Araujo et al. (2013) ${ }^{18}$ também relataram que os alunos que participaram de um projeto de extensão, no qual eram prestados atendimentos à comunidade e realizadas atividades de promoção de saúde (envolvendo 12 alunos de Odontologia de uma universidade de São Paulo), relataram perceber um diferencial em comparação aos que não participaram, e seus professores observaram que eles se tornaram mais confiantes em realizar procedimentos clínicos e na tomada de decisões, atuando de uma forma mais humana e crítica $^{18}$

As atividades de caráter voluntário que são proporcionadas aos acadêmicos da área de saúde por meio dos projetos de extensão são de grande valia para a formação de um futuro profissional de excelência. $\mathrm{O}$ acadêmico consegue pôr em pratica o que aprendeu em sala de aula e adquire novos conhecimentos, tanto acadêmicos, quanto sobre a comunidade ao seu redor, o que o faz com que conheça a realidade social e os problemas da população, formando assim um cirurgião-dentista que irá assistir seus pacientes de forma integral e humanizada, que tem maior sensibilidade social e cultural, e consegue assim realizar intervenções de prevenção e promoção de saúde bem sucedidas ${ }^{6,15}$. Infelizmente, ainda é necessário enfrentar dificuldades acerca da percepção de discentes e docentes sobre as atividades extramuros, as quais são propostas, geralmente, pela disciplina de Saúde Coletiva, e são vistas como atividades acessórias, com menor valor para a formação profissional ${ }^{19,12}$. 
Oliveira et al. $(2016)^{20}$ discutiram as dificuldades no desenvolvimento de projetos de extensão universitária pelos acadêmicos e verificaram que as questões de preparação, comportamento, continuidade do projeto e falta de recursos materiais são limitantes e demonstram a menor importância dada a estas atividades em comparação a outras áreas da formação técnica profissional. Muito importante é reconhecer que a ação de extensão não se baseia somente em sair dos muros da academia e ir a uma comunidade sem ao menos compreender a sua realidade. É essencial que o estudante receba uma capacitação que lhe forneça conhecimento acerca das características, demandas e especificidades do público com o qual atuará. Sendo assim, o enfrentamento de situações comuns à sociedade exige preparo técnico e profissional e, mais ainda, a capacidade de lidar com a complexidade própria do ser humano ${ }^{21}$.

Atualmente as universidades passam por um momento de mudança, que derivam dos questionamentos sobre seu papel na geração de conhecimento, sendo assim um desafio formar profissionais que estão preparados para lidar com as necessidades sociais. Com isso, deve-se proporcionar aos alunos atividades que os tornem profissionais capacitados, que saibam trabalhar em equipes multiprofissionais, saibam comunicar-se adequadamente, sejam confiantes, tomem decisões frente a dificuldades, e principalmente que saibam lidar com a comunidade onde irão trabalhar, entendendo suas dificuldades e se tornando cirurgiões-dentistas mais humanizados. Porém, conforme discutido por Biscarde et al. (2014) $)^{8}$, essas características dificilmente são obtidas com o método de ensino tradicional, no qual os ensinamentos vão sendo passados ao decorrer do curso, praticados separadamente em clínicas específicas para cada disciplina, dificultando ao acadêmico relacionar os conteúdos aprendidos, levando-o a usar seus conhecimentos de forma fragmentada e não atendendo os indivíduos com uma visão integral. O problema das práticas realizadas nas clínicas intramuros nas universidades é que podem fazer com que o universitário tenha uma visão alterada da rede de atendimentos do sistema de saúde, sendo assim, é necessário que o aluno aprenda em espaços diversificados $^{8,}$ o que contribui para uma interação entre a sociedade e a universidade, permitindo assim, uma troca de saberes e experiências entre a população, os acadêmicos e os professores ${ }^{6,8,15}$.

Os cursos de Odontologia deveriam contemplar tanto as práticas reabilitadoras quanto as de promoção da saúde por meio de ações extensionistas $^{6}$, nas quais se constroem novos e diferentes saberes, aproximando a academia da sociedade ${ }^{22}$.

\section{CONCLUSÕES}

A extensão universitária contribui significativamente para a formação de cirurgiõesdentistas preparados para atuar no campo de trabalho, que saibam atender às necessidades da população, já que promove uma efetiva interação do estudante com a comunidade, fazendo com que este se torne, futuramente, um profissional completo, que vê seus pacientes de forma integral, e que compreende de forma mais ampla o complexo processo saúde-doença. Além de beneficiar aos alunos, a extensão possibilita à população o acesso a conhecimentos importantes sobre saúde, em uma troca de saberes que contribui para o crescimento de ambas as partes.

\section{ABSTRACT \\ Importance of university extension in the professional training of dental surgeons}

Universities have transformed over time. The main focus of teaching started to expand with research and extension activities. Extension courses have been increasingly introduced in higher education institutions, participating in the triad that sustains university education. This literature review aimed to show the importance 
of university extension in the professional training of dental surgeons and society. The strategy for searching articles was based on keywords related to dentistry and university extension, in electronic databases, from August to October 2019. In different communities, the practice of extension strengthens the understanding of students about the healthdisease process and allows knowing the economic and social realities of different scenarios, experiencing the public health care network, and participating in the provision of services to the population, which makes students more humanized and able to promote health to their communities. University extension plays an important role in the professional training of dental surgeons, helping them to work competently assisting in the diverse needs of the population, stimulating health promotion and information to the general population who also benefits from these activities, and strengthening the commitment of the university to society.

Descriptors: Community Extension. Community Dentistry. Continuing Education.

\section{REFERÊNCIAS}

1. Coelho GC. O papel pedagógico da extensão universitária. Em Ext. 2014; 13,(2):11-24.

2. Pivetta HFP, Backes DS, Carpes A, Battistel ALHT, Marchiori M. Ensino, pesquisa e extensão universitária: em busca de uma integração efetiva. Linhas Críticas. 2010; 16(31):377-90.

3. Moimaz SAS, Gomes AMP, Bordin D, Garbin, CAS, Saliba NA. Extensão universitária como ferramenta geradora de ensino-aprendizagem e produtora de pesquisa. Revista Conexão UEPG. 2015; 11(2):140-149.

4. Brasil. Ministério da Educação. Conselho Nacional de Educação. Câmara de Educação Superior. Resolução no. 7, de 18 de dezembro de 2018. [Acesso em 24 jul. 2021. Disponível em: http://portal.mec.gov.br/ index.php?option=com_docman\&view $=$ do wnload\&alias $=104251-$ rces007-18\&catego

ry_slug=dezembro-2018-pdf\&Itemid=30192.

5. Emmi DT, Silva DMC, Barroso RFF. Experiência do ensino integrado ao serviço para formação em Saúde: percepção de alunos e egressos de Odontologia. Interface. 2018; 22(64):223-36.

6. Pereira SM, Mialhe FL, Pereira LJ, Soares MF, Tagliaferro PS, Meneghim MC, Pereira AC. Extensão universitária e trabalho voluntário na formação do acadêmico em Odontologia. Arq Odontol. 2011; 47(2): 95103.

7. Ponte CIRV, Torres MAR, Machado CLB, Manfroi WC. A extensão universitária na Famed/UFRGS: cenário de formação profissional. Rev Bras Educ Méd. 2009; 33(4):527-34.

8. Biscarde DGS, Santos MP, Silva LB. Formação em saúde, extensão universitária e Sistema Único de Saúde (SUS): conexões necessárias entre conhecimento e intervenção centradas na realidade $\mathrm{e}$ repercussões no processo formativo. Interface. 2014; 18(48):177-86.

9. Fórum de Pró-Reitores de Extensão das Universidades Públicas Brasileiras e pela Secretaria de Educação Superior do Ministério da Educação e do Desporto. Plano Nacional de Extensão Universitária. 2000-2001:17.

10. Hennington ÉA. Acolhimento como prática interdisciplinar num programa de extensão universitária. Cad Saúde Pública. 2005; 21(1):256-65.

11. Rodrigues ALL, Prata MS, Batalha TBS, Costa CLN, Neto IFP. Contribuições da extensão universitária na sociedade. Cad Grad Ciênc Hum Soc. 2013; 1(16):141-8.

12. Borato A, Pereira MVS, Bordin D, Martins AS, Fadel CB. Valoração das práticas de ensino, pesquisa e extensão entre 
concluintes de Odontologia. Rev Abeno. 2018; 18(1):103-15.

13. Brasil. Ministério da Educação. Conselho Nacional de Educação. Câmara de Educação Superior. Resolução no. 3, de 19 de fevereiro de 2002. [Acesso em 24 jul. 2021. Disponível em: http://portal.mec.gov.br lindex.php?option=com_docman\&view=do $\underline{\text { wnload \&alias }=192671-\text { rces003-02\&catego }}$ ry_slug=junho-2021-pdf\&Itemid=30192.

14. Cruz BPA, Melo WS, Malafaia FCB, Tenório FG. Extensão universitária e responsabilidade social: 20 anos de experiência de uma instituição de ensino superior. RGSA, 2011; 5(3):3-16.

15. Fadel CB, Bordin D, Kuhn E, Martins LD. O impacto da extensão universitária sobre a formação acadêmica em odontologia. Com Saúde Educ. 2013; 17(47):937-46.

16. Falcão EF. Vivência em comunidade outra forma de ensino. Editora da UFPB, 2014. 208p. ISBN: 978-85-237-0856-6

17. Weiler L, Haddock S, Zimmerman TS, Krafchick J, Henry K, Rudisill S. Benefits derived by college students from mentoring at-risk youth in a service-learning course. Am J Commun Psychol. 2013; 52(3-4):236248.

18. Araujo RM, Feitosa FA. Articulando o ensino de graduação em odontologia com a extensão universitária. Rev Ciênc Ext. 2013; 9(3):115-24.
19. Toassi RFC, Baumgarten A, Warmling CM, Rossoni E, Rosa AR, Slavutzky SMB. Teaching at primary healthcare services within the Brazilian National Health System (SUS) in Brazilian healthcare professionals' training. Interface. 2013; 17(45):385-92.

20. Oliveira FLB, Almeida Júnior JJ, Silva MLP. Percepção dos acadêmicos em relação às dificuldades no desenvolvimento de projetos de extensão universitária. Rev Ciênc Ext. 2016; 12(2):18-25.

21. Ribeiro RMC. A extensão universitária como indicativo de responsabilidade social. Diálogo. 2011; 15(1):81-8.

22. Divino AEA, Costa CLNA, Oliveira CEL, Costa CAC, Neta HRS, Campos LS, et. al. A extensão universitária quebrando barreiras. Cad Grad Ciênc Hum Soc. 2013; 1(16):135140.

\section{Correspondência para:}

Gabriela Pizzolatto

e-mail: gabrielapizzolatto@gmail.com

Rua Moron, 1427

99010-032 Passo Fundo/RS 